\title{
Million Units per Milliliter
}

National Cancer Institute

\section{Source}

National Cancer Institute. Million Units per Milliliter. NCI Thesaurus. Code C67428.

An arbitrary unit of substance concentration equal to the concentration at which one

milliliter of mixture contains one million units of a substance. 